Cómo citar este artículo: Hernández-Fernández, N. J., Zumalacárregui-de Cárdenas, L., \& Pérez-Ones, O. (2020). Simulación de condiciones de operaciones y fluidos de trabajo para ciclos Rankine orgánicos. Rev.investig.desarro.innov., 10 (2), 349-358.

\title{
Simulación de condiciones de operación y fluidos de trabajo para ciclos Rankine orgánicos
}

\section{Simulation of operating conditions and working fluids for organic Rankine cycles}

\author{
Nerio José Hernández-Fernández ${ }^{1}$ \\ Lourdes Zumalacárregui-de Cárdenas ${ }^{2}$ \\ Osney Pérez-Ones ${ }^{3}$
}

Recibido: abril 05 de 2019 Aceptado: septiembre 16 de 2019

\section{Resumen}

En el presente trabajo se comparan condiciones de operación para diferentes fluidos en ciclos de Rankine orgánicos, que utilicen energías de bajo grado. Los fluidos estudiados fueron: R152a (1,1-difluroetano), R-290 (propano), R-600a (2-metilpropano o isobutano), R-744 (dióxido de carbono), y R-1270 (propileno). Se realizó la simulación del ciclo de Rankine orgánico y de sus modificaciones: ciclo con recalentamiento intermedio, ciclo de regeneración y ciclo con recalentamiento y regeneración. Se usó el simulador de procesos HYSYS v3.2. Los mejores resultados se alcanzaron para el ciclo con regeneración, aunque los rendimientos térmicos fueron inferiores al $10 \%$. Se simularon condiciones de operación que permitieran superar este valor, lo cual se logró con el refrigerante R-600a en el ciclo Rankine con regeneración, que opere entre $3500 \mathrm{kPa}$ y $150^{\circ} \mathrm{C}$ a la entrada de la turbina, y $700 \mathrm{kPa}$ en el condensador. Con ello se obtiene un rendimiento térmico de $15,33 \%$ y se producen $7,11 \mathrm{~kW}$ de potencia eléctrica.

Palabras clave: ciclos de potencia, ciclo Rankine orgánico, fluidos orgánicos, simulador de procesos.

\begin{abstract}
Operating conditions for different fluids in Organic Rankine Cycles using low grade energies were compared. The following fluids were used: R152a (1,1-difluroethane), R-290 (propane), R-600a (2-methylpropane or isobutane), R-744 $\left(\mathrm{CO}_{2}\right)$ y R-1270 (propylene). Simulation with HYSYS v3.2 was used to support the research. Organic Rankine cycle was studied. Also, the following modifications were studied: reheating, regeneration and reheating-regeneration. Regeneration cycle showed the best results, however, thermal efficiencies were lower than 10\%. Operating conditions were simulated in order to increase this value. It was determined that the fluid that presented the best behavior was the $\mathrm{R}-600$ a refrigerant, using a Rankine with regeneration cycle operating between $3500 \mathrm{kPa}$ and $150{ }^{\circ} \mathrm{C}$ at the turbine entrance and $700 \mathrm{kPa}$ in the condenser. A thermal efficiency of $15.33 \%$ was obtained and $7.11 \mathrm{~kW}$ of electric power can be produced.
\end{abstract}

Keywords: power cycle, organic Rankine cycle, organic fluids, process simulation.

1 Ingeniero Químico, Máster en Ciencias, ISS International-Integrated Service Solutions, Ciudad Panamá, Panamá. E-mail: nerio1410@gmail.com ORCID: 0000-0001-7724-2583

2 Ingeniera Química, Doctora en Ciencias Técnicas, Universidad Tecnológica de La Habana "José Antonio Echeverría", CUJAE, La Habana, Cuba. E-mail: lourdes@quimica.cujae.edu.cu. ORCID:0000-0001-6921-737X

3 Ingeniero Químico, Doctor en Ciencias Técnicas, Universidad Tecnológica de La Habana "José Antonio Echeverría", CUJAE, La Habana, Cuba. E-mail: osney@quimica.cujae.edu.cu. ORCID: 0000-0002-0366-0317 


\section{Introducción}

El consumo cada vez mayor a nivel industrial y doméstico de la energía eléctrica, ha llevado a la utilización excesiva de fuentes de generación de energía. Estas causan un deterioro y consumo de recursos naturales provocando un impacto nocivo al medio ambiente, así como efectos indeseables en la atmósfera del planeta, producto de la emanación de gases contaminantes (Bautista-Ruiz, Díaz-Lagos \& Martínez-Ovalle, 2017). Tal problemática obliga a distintos países a replantearse sus políticas en cuanto a generación eléctrica, con el objetivo de encontrar maneras más adecuadas para su producción (Vásquez, 2013).

Para realizar los procesos de recuperación energética a partir de calores residuales de procesos industriales, es necesario recurrir a las tecnologías de generación a partir de fuentes de calor de baja y media temperatura (menores a $250-300^{\circ} \mathrm{C}$ ). Estas no solo incluyen fuentes de calor residuales, sino que también abarcan las de energía renovable, como: la geotérmica, la solar y la aportada en la combustión de biomasa. La tecnología con más alto desarrollo en ese tema es el ciclo de Rankine orgánico (ORC, por sus siglas en inglés), con el cual se puede generar trabajo mecánico con una fuente de calor desde los $80{ }^{\circ} \mathrm{C}$ en dependencia del fluido de trabajo, pero con eficiencias termodinámicas bajas (Mikielewicz, 2010; Desintec Ingeniería, 2009).

Entre los compuestos orgánicos que pueden utilizarse en el ciclo de Rankine, se encuentran los refrigerantes, que poseen características físicas y químicas que atraen su interés. Entre ellas se pueden mencionar: la baja temperatura de ebullición, lo cual conduce a un menor consumo energético; la posibilidad de encontrar algunos de baja toxicidad, con lo que no se contribuiría a la contaminación ambiental ante posibles fugas; y el fácil acceso a ellos (Borshanpour \& Wallace, 2011).

Cuando se trata de recuperar la energía de bajo grado, el líquido generalmente tiene una temperatura de ebullición más baja que el agua, siendo los refrigerantes e hidrocarburos compuestos de uso común para esta aplicación. Las características más importantes de un buen fluido de trabajo orgánico son: alta entalpía de vaporización y densidad para absorber más energía del evaporador y reducir el flujo requerido; el tamaño del equipamiento; baja toxicidad; buena compatibilidad y estabilidad química en comparación con otros materiales; bajo impacto ambiental, considerando como los principales parámetros para esta selección el potencial de agotamiento del ozono y el potencial de calentamiento global; seguridad, debiendo ser el líquido poco inflamable, poco corrosivo y con bajo potencial para la descomposición y buena disponibilidad y bajo costo (Chen, Yogi \& Stefanakos, 2010).

Otro rasgo característico importante en la selección de un fluido, es la forma de la curva de puntos de rocío. La pendiente de la curva de saturación del fluido de trabajo en un diagrama temperatura-entropía (T-s), puede ser: positiva, negativa o vertical. Estos fluidos se conocen como: fluidos secos, húmedos e isentrópicos, respectivamente. Los fluidos húmedos, como el agua, requieren del sobrecalentamiento del vapor, mientras que los secos o isentrópicos, no lo requieren (Chen et al., 2010).

El ORC presenta varias ventajas sobre el ciclo tradicional de vapor de agua: la caldera trabaja a menores temperaturas (menos de $300{ }^{\circ} \mathrm{C}$ ), en cambio los ciclos de vapor trabajan a mayores temperaturas (hasta $450{ }^{\circ} \mathrm{C}$ ), para evitar la formación de gotas durante la expansión; menores presiones, menor costo de instalación y más seguridad (Quoilin, Van den Broek, Declaye, Dewallef \& Lemort, 2013). En este sentido, los fluidos refrigerantes resultan prometedores para los ciclos ORC, especialmente en relación a la baja toxicidad de algunos de ellos.

Entre los fluidos que se han usado en ciclos de Rankine orgánicos, están: R123 $\left(\mathrm{CHCl}_{2} \mathrm{CF}_{3}\right)$ (Majo, Chamra, Srinivasan, \& Somayaji, 2008); R134a 
$\left(\mathrm{C}_{2} \mathrm{H}_{2} \mathrm{~F}_{4}\right), \mathrm{R} 141 \mathrm{~b}\left(\mathrm{C}_{2} \mathrm{H}_{3} \mathrm{Cl}{ }_{2} \mathrm{~F}\right), \mathrm{R} 5050\left(\mathrm{CF}_{3}\left(\mathrm{CF}_{2}\right) 3 \mathrm{CF}_{3}\right)$ (Madhawa, Golubovic, Worek \& Ikegami, 2007); isobuteno $\left(\left(\mathrm{CH}_{3}\right)_{2} \mathrm{C}=\mathrm{CH}_{2}\right)$ (Majo et al., 2008); $\mathrm{R} 245 \mathrm{fa}$ (Wang et al., 2010); e hidrocarburos aromáticos (Fankam, Papadakis, Lambrinos \& Frangoudakis, 2009).

Algunos ejemplos de aplicación de los ORC usan como fuente de calor energía geotérmica (Borshanpour \& Wallace, 2011), y energía solar (Pei, Li \& Ji, 2010); Vélez, Segovia, Martín, \& Antolí, 2012), con eficiencias de: $8,2 \%, 7-9 \%$ y $4 \%$, respectivamente. Fontalvo-Lascano y colaboradores (2018), evaluaron la rentabilidad de la cogeneración de un ORC simple acoplado, mediante un circuito intermedio de aceite térmico, a los gases de escape del motor QSV91G, operando a una carga parcial del $75 \%$. Los fluidos de trabajo estudiados fueron: R245fa, R1233zd(E) y R1234ze(Z). Se alcanzaron eficiencias térmicas entre 14 y $16 \%$, para temperaturas del aceite entre 200 y $250^{\circ} \mathrm{C}$.

El estudio de los ciclos de potencia termodinámicos, usualmente se realiza empleando simuladores (Salazar-Pereira et al., 2017). El modelo de un ciclo de Rankine usando el simulador de procesos HYSYS, se aplicó en plantas de ciclo combinado, con buenos resultados y errores de estimación inferiores al 5\% (Lopéz, Montes de Oca, Díaz, Tápanez \& Domínguez, 2009; Cano, 2013). Otro ejemplo es el de Giuffrida y Pezzuto (2017), que simularon la operación de un ciclo Rankine orgánico que utiliza un expansor de $2 \mathrm{~kW}$. Estos autores muestran que cuando se usa R1234ze (Z) o R1233zd (E) como sustitutos del R245fa original, el rendimiento térmico aumenta; sin embargo, las eficiencias térmicas no rebasan el $10 \%$ para temperaturas entre 80 y $120^{\circ} \mathrm{C}$.

La viabilidad termodinámica de un ORC para tres fuentes renovables diferentes: energía geotérmica, energía solar y biomasa, se estudió mediante simulación del ciclo ORC, en el programa Aspen HYSYS v3.2 (López., 2013). En el caso de la fuente térmica biomasa, los gases exhaustos de la caldera de biomasa calientan un aceite sintético hasta $350^{\circ} \mathrm{C}$, y este le cede energía al ORC. El autor trabajó con ciclopentano y octametiltetrasiloxano, a temperaturas de $280-350^{\circ} \mathrm{C}$.

En este trabajo se evaluaron, con auxilio de la simulación, las condiciones de operación requeridas para producir electricidad según un ciclo de Rankine orgánico, trabajando con fluidos puros, y en los que sealcance un rendimiento térmico mayor del $10 \%$. Se espera que los resultados, a futuro, permitan llevar la energía eléctrica a lugares que, por su situación geográfica, presentan dificultad para recibir un servicio eléctrico convencional.

\section{Materiales y métodos}

\subsection{Fluidos de trabajo}

Del análisis de las propiedades físicas, químicas y medioambientales de la literatura (Air Liquide, 2014; National Refrigerants, 2014; Renedo-Estébanes, 2014), se seleccionaron para este estudio los fluidos: R152a (1,1-difluroetano), R-290 (propano), R-600a (2-metilpropano o isobutano), R-744 (dióxido de carbono) y R-1270 (propileno). Para estos fluidos, el agotamiento del ozono toma valor 0 y el potencial de calentamiento global, aunque mayor que 1, valor para el R-744, se mantienen inferiores a 20, de donde se pueden considerar bajos (Borshanpour \& Wallace, 2011).

Si bien se recomienda que los fluidos sean inflamables, no corrosivos y no tóxicos, no siempre es posible satisfacer todas las características. Por ejemplo, aunque sean inflamables, si no existen fuentes de ignición cercanas, se trabaja a temperaturas por debajo de las de autoignición y se toman las medidas de seguridad establecidas, este efecto puede minimizarse.

\subsection{Ciclo Rankine convencional y simulación}

En este trabajo se utilizó el simulador HYSYS v3.2. Para los fluidos orgánicos se utilizó el paquete de propiedades de Zudkevitch-Joffe, ya que evalúa 
las propiedades físicas de fluidos que contienen hidrógeno $(\mathrm{H})$ y derivados del petróleo $(\mathrm{CH})$ en el equilibrio líquido-vapor (ELV) y había sido validado para algunos fluidos similares (Olazabal, 2014).

Se simuló el sistema de cogeneración analizado por Olazabal (2014), cuyo esquema se representa en la figura 1, operando con un ciclo de Rankine convencional. En este caso, el agua de enfriamiento que se calienta en el condensador se utiliza como fuente térmica para uso doméstico. Los componentes introducidos fueron: aceite térmico el 1,2 diclorobenceno $\left(\mathrm{C}_{6} \mathrm{H}_{4} \mathrm{Cl}_{2}\right)$; como fluido de enfriamiento en el condensador el agua, y como fluido de trabajo en el ciclo: R-152a para el primer caso, R-290 para el segundo caso, R-600a para el tercer caso, R-744 para el cuarto caso y el R-1270 para el quinto caso. El esquema de la figura 1, se utilizó para la simulación y se validó su adecuación por Olazabal (2014).

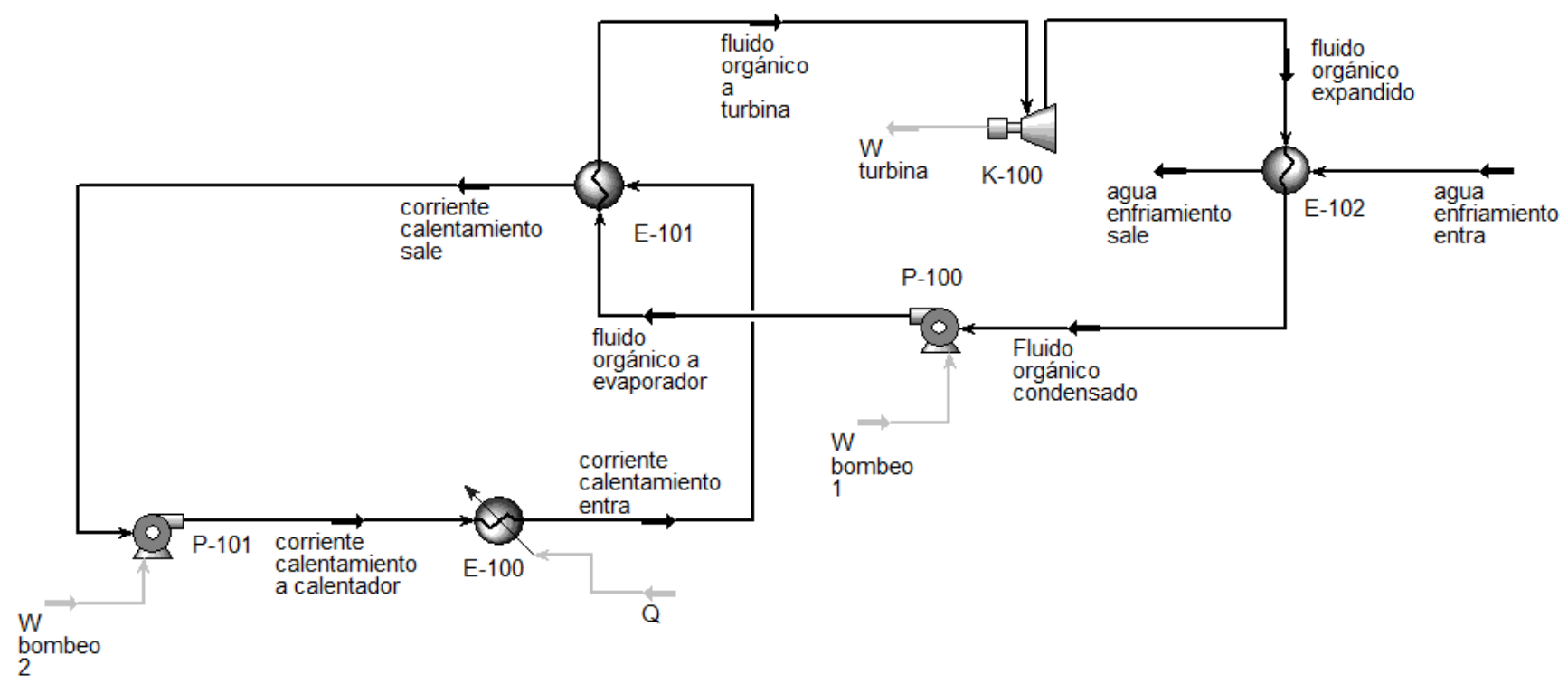

Figura 1. Esquema de simulación del ciclo operando como Rankine convencional.

Cabe destacar que hubo que realizar modificaciones en el diagrama de flujo de proceso para el refrigerante R-744 (dióxido de carbono), al tener un punto de ebullición por debajo de los $0{ }^{\circ} \mathrm{C}$, lo que impidió obtener un resultado convergente con el esquema original. La modificación consistió en reemplazar el equipo de intercambio de calor (E-102), por un condensador, a fin de evitar el cruce de temperatura entre el fluido orgánico condensado y el agua de enfriamiento. De esta forma se obtiene la energía necesaria a extraer en el condensador, la cual debe ser absorbida por un fluido que sea capaz de operar a los bajos niveles de temperatura para este refrigerante. En este caso se requeriría un fluido con una temperatura por debajo de $0^{\circ} \mathrm{C}$, en correspondencia con la temperatura de saturación a $2000 \mathrm{kPa}$ de los fluidos estudiados. Las variables de decisión utilizadas para todos los fluidos, se presentan en la Tabla 1. 
Tabla 1. Variables de decisión utilizadas para todos los fluidos.

\begin{tabular}{ll}
\hline Variables & Valor \\
\hline $\mathrm{m}(\mathrm{kg} / \mathrm{s})$ & 504 \\
T entrada a la turbina $\left({ }^{\circ} \mathrm{C}\right)$ & 150 \\
$\mathrm{P}$ entrada a la turbina $(\mathrm{kPa})$ & 3500 \\
$\mathrm{P}$ salida de la turbina $(\mathrm{kPa})$ & 2000 \\
$\Delta \mathrm{P}$ en el evaporador $(\mathrm{kPa})$ & 0,44 \\
$\Delta \mathrm{P}$ en el condensador $(\mathrm{kPa})$ & 3,00 \\
$\mathrm{~T}$ de entrada del agua al condensador $\left({ }^{\circ} \mathrm{C}\right)$ & 20 \\
$\mathrm{~T}$ de salida del agua del condensador $\left({ }^{\circ} \mathrm{C}\right)$ & 45 \\
T de entrada de la corriente de calentamiento al evaporador $\left({ }^{\circ} \mathrm{C}\right)$ & 250 \\
T de salida de la corriente de calentamiento al evaporador $\left({ }^{\circ} \mathrm{C}\right)$ & 230 \\
Eficiencia de la bomba $(\%)$ & 85 \\
Eficiencia de la turbina $(\%)$ & 80 \\
\hline
\end{tabular}

\section{Resultados y discusión}

3.1 Simulación según el ciclo de Rankine convencional

En la Tabla 2, se evidencia que el fluido de trabajo con el que se alcanzó un mejor rendimiento térmico fue el refrigerante R-744, con $7,65 \%$ de rendimiento y una capacidad de generación de energía en la turbina de 4,8 kW. Sin embargo, el inconveniente de este refrigerante es que requiere de un medio de enfriamiento a temperaturas inferiores a $0{ }^{\circ} \mathrm{C}$, situación no común a menos que se disponga de un ciclo de refrigeración. En consecuencia, se seleccionó el refrigerante $\mathrm{R}-1270$, con $6,91 \%$ de rendimiento térmico y la producción de 4,56 kW en la turbina. No obstante, con este esquema no se alcanza más del $10 \%$ de rendimiento térmico deseado.

Tabla 2. Resultados de la simulación según el ciclo de Rankine convencional.

\begin{tabular}{llllll}
\hline Parámetros & Fluidos orgánicos \\
& R-152a & R-290 & R-600a & R-744 & R-1270 \\
Flujo molar a la entrada turbina K-100 $(\mathrm{kmol} / \mathrm{h})$ & 7,63 & 11,43 & 8,67 & 11,45 & 12,00 \\
Temperatura a la salida de la turbina $\left({ }^{\circ} \mathrm{C}\right)$ & 121,5 & 125,9 & 124,1 & $-31,87$ & 123,1 \\
Trabajo producido en la turbina K-100 $(\mathrm{kW})$ & 2,724 & 4,257 & 2,63 & 4,77 & 4,56 \\
Potencia absorbida en evaporador E-101 $(\mathrm{kW})$ & 42,0 & 64,2 & 40,5 & 62,4 & 66,0 \\
Rendimiento térmico $(\%)$ & 6,49 & 6,63 & 6,50 & 7,65 & 6,91 \\
\hline
\end{tabular}

Se estudiaron varias modificaciones al ciclo Rankine con la finalidad de elevar el rendimiento térmico obtenido, a saber: ciclo Rankine con regeneración, recalentamiento, y la combinación de recalentamiento y regeneración, las cuales se presentan a continuación.

\section{Ciclo Rankine con regeneración}

En la figura 2 se presenta el esquema de simulación del ciclo con regeneración. La presión a la salida de la turbina de alta presión, fue de 2700 $\mathrm{kPa}$. En la Tabla 3 se presentan los resultados de la simulación. Se aprecia que con esta configuración 
el rendimiento térmico es superior al del ciclo de Rankine convencional, ya que con el regenerador se precalienta el fluido de trabajo a la entrada del evaporador, utilizando parte de la energía calorífica del fluido que sale del expansor. Esto permite obtener una potencia similar, pero demandando una energía térmica menor en el evaporador.

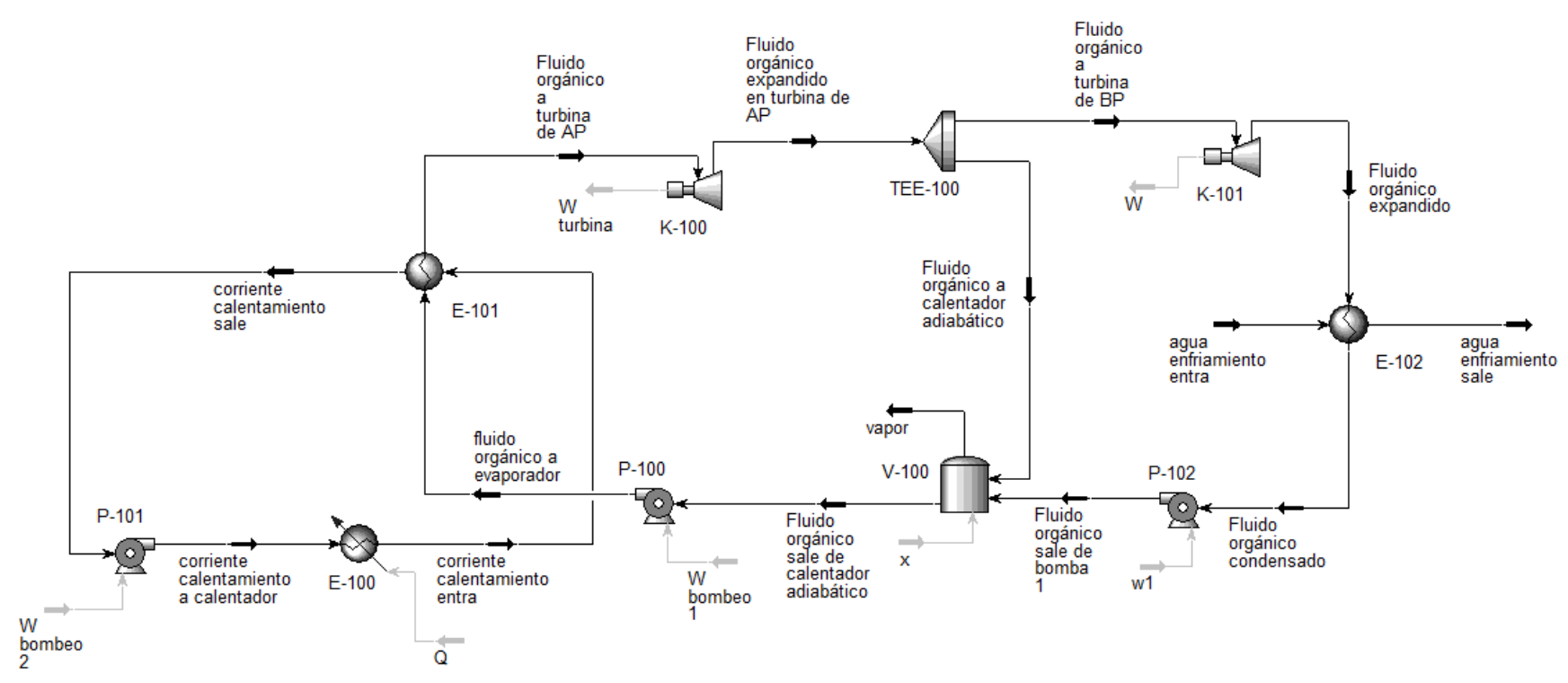

Figura 2. Esquema de simulación del ciclo de regeneración.

Tabla 3. Resultados de la simulación según los ciclos de Rankine modificados.

Parámetros

Trabajo producido en las turbinas (kW)

Potencia absorbida en evaporador E-101 (kW)

Rendimiento térmico (\%)

Ciclo Rankine con recalentamiento

Trabajo producido en las turbinas (kW)

Potencia absorbida en evaporador E-101 y recalentador E-103 (kW)

Rendimiento térmico (\%)

Ciclo Rankine con regeneración y recalentamiento

Trabajo producido en las turbinas (kW)

Potencia absorbida en evaporador E-101 y recalentador E-103 (kW)

Rendimiento térmico (\%)
Fluidos orgánicos

$\begin{array}{lllll}R-152 a & R-290 & R-600 a & R-744 & \text { R-1270 }\end{array}$

$2,55 \quad 3,97 \quad 2,29 \quad 4,65 \quad 4,30$

$36,67 \quad 55,91 \quad 31,31 \quad 59,00 \quad 58,88$

$6,95 \quad 7,10 \quad 7,32 \quad 7,88 \quad 7,30$

$2,83 \quad 4,37 \quad 2,76 \quad 4,48 \quad 4,69$

$\begin{array}{lllll}44,77 & 67,91 & 45,26 & 64,95 & 69,71\end{array}$

$6,30 \quad 6,43 \quad 6,10 \quad 6,91 \quad 6,72$

$2,64 \quad 4,08 \quad 2,43 \quad 4,79 \quad 4,42$

$39,47 \quad 59,97 \quad 36,17 \quad 61,69 \quad 59,24$

$\begin{array}{rrrrr}6,69 & 6,80 & 6,74 & 7,76 & 7,46\end{array}$

El fluido orgánico con mayor rendimiento térmico, es el refrigerante R-744, con $7,88 \%$ y una potencia eléctrica de 4,65 kW; sin embargo, con el R-1270 se logran resultados similares, usando agua como medio de enfriamiento. No obstante, no se alcanza más del $10 \%$ de rendimiento térmico deseado. 


\section{Ciclo Rankine con recalentamiento intermedio}

En la figura 3 se presenta el esquema del ciclo con recalentamiento. La presión a la salida de la turbina de alta presión, fue de $2700 \mathrm{kPa}$ y la temperatura de salida del recalentador $149^{\circ} \mathrm{C}$. Los resultados de la simulación se presentan en la Tabla 3. Con esta alternativa no se logran rendimientos térmicos superiores a los del ciclo de Rankine convencional. Esto se explica porque para el valor de presión intermedia a la que se realiza el recalentamiento, se obtienen incrementos en el trabajo producido inferiores a los aumentos de energía absorbida, en donde el cociente, que corresponde al rendimiento térmico, puede ser menor.

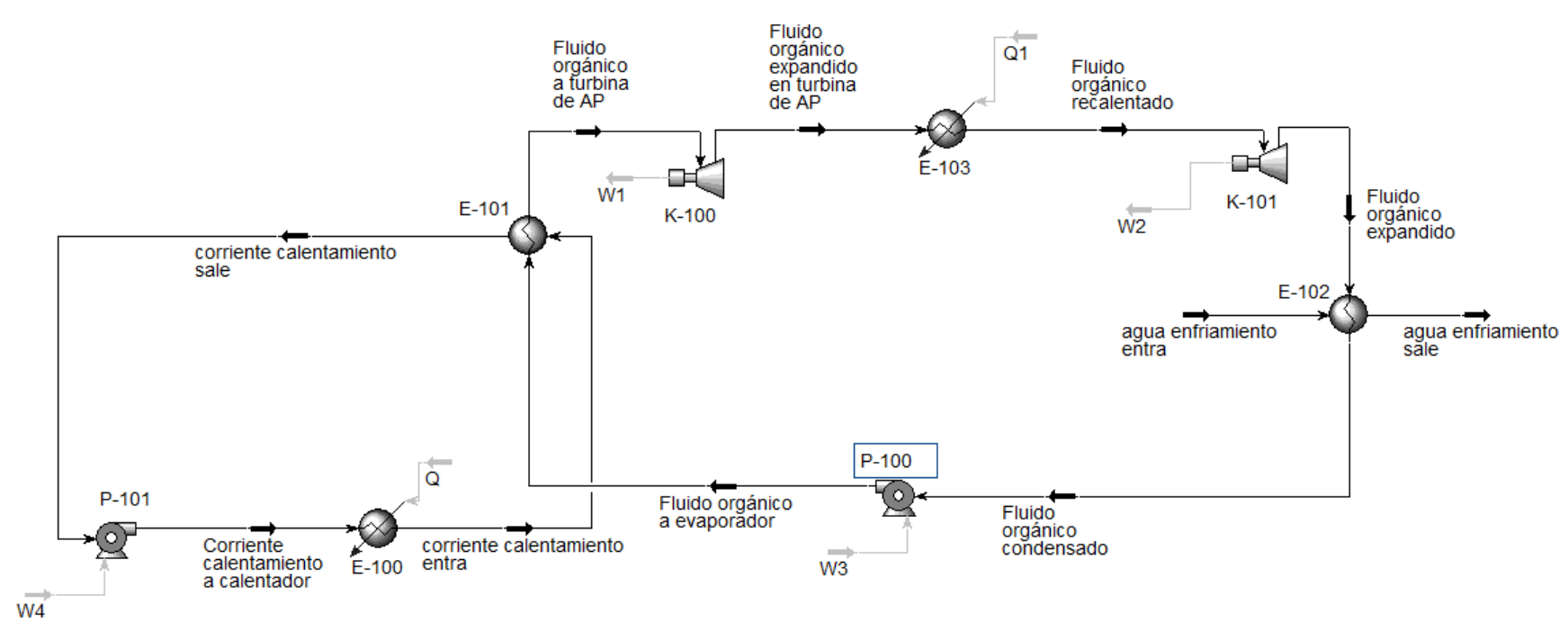

Figura 3. Esquema de simulación del ciclo con recalentamiento intermedio.

\section{Ciclo Rankine con recalentamiento intermedio y regeneración}

En la simulación se combinan los dos casos anteriores y sus resultados se presentan en la Tabla 3. Para el ciclo con recalentamiento intermedio y regeneración, el fluido con mayor rendimiento térmico fue el R-744, con un 7,76\% y una generación de potencia eléctrica en las turbinas de 4,79 $\mathrm{kW}$. Al seleccionar un refrigerante con posibilidad de enfriarse con agua, el mejor es el R-1270, con $7,46 \%$ de rendimiento térmico y potencia producida de 4,42 kW. Como se observa, con este ciclo se logran niveles de rendimiento superiores a los del Rankine convencional y similares a los del ciclo con regeneración. No obstante, los niveles de rendimiento térmico no alcanzan el $10 \%$ deseado.

\subsection{Casos de estudio}

En esta investigación se desea un rendimiento térmico superior al $10 \%$, valor obtenido por otros investigadores en trabajos con ORC (Campos et al., 2013). En este sentido, se desarrollaron dos casos de estudio para determinar si existen condiciones en que se sobrepase el valor deseado de rendimiento térmico, utilizando un ciclo de Rankine con regeneración. Esto debido a que es el de mejores rendimientos y menor complejidad, en relación con el Rankine con recalentamiento y regeneración.

Se estima que para alcanzar valores superiores al $15 \%$, habría que explorar utilizando el R-600a. Esto debido a que, para las condiciones de salida de la turbina, presenta una temperatura de $100,7^{\circ} \mathrm{C}$, existiendo un amplio gradiente de temperatura 
entre este valor y la temperatura del medio de enfriamiento que se está usando, agua entre $20^{\circ} \mathrm{C}$ y $45^{\circ} \mathrm{C}$. Este valor es la mayor temperatura de entre todos los fluidos estudiados.

Caso 1: influencia de la presión de entrada a la turbina en la potencia generada

La variable independiente fue la presión a la entrada de la turbina de alta presión $(\mathrm{kPa}), y$ se varió entre $3400 \mathrm{kPa}$ y $4000 \mathrm{kPa}$. En la Tabla 4 se aprecia que, a medida que la presión a la entrada de la turbina aumenta, la potencia en la turbina de alta aumenta, mientras que disminuye la de la turbina de baja presión. A partir de los valores obtenidos se calcularon los rendimientos térmicos, verificando que con esta modificación no se alcanzan valores de rendimientos superiores al $10 \%$.

Tabla 4. Variación de la potencia generada y el rendimiento térmico con la presión a la entrada de la turbina.

\begin{tabular}{lll}
\hline Presión $(\mathbf{k P a})$ & Potencia generada $(\mathbf{k W})$ & Rendimiento térmico $(\%)$ \\
\hline 3400 & 2,21 & 6,94 \\
3600 & 2,37 & 7,77 \\
3800 & 2,48 & 8,56 \\
4000 & 2,53 & 9,31 \\
\hline
\end{tabular}

En la tabla 4 se aprecia que, aún cuando los niveles de rendimiento pueden resultar más altos (9,31\%), se requiere aumentar la presión de trabajo, manteniendo un nivel de producción de potencia eléctrica bajo. Por consiguiente, no es una alternativa favorable.
Caso 2: influencia de la presión a la salida de la turbina en la potencia generada

La presión a la salida del condensador, se modificó entre $700 \mathrm{kPa}$ y $1900 \mathrm{kPa}$. En la Tabla 5 se presenta un resumen de estos resultados.

Tabla 5. Variación de la potencia y el rendimiento térmico con la presión a la salida de la turbina.

\begin{tabular}{lll}
\hline Presión $\mathbf{( k P a )}$ & Potencia generada $(\mathbf{k W})$ & Rendimiento térmico $(\%)$ \\
\hline 700 & 7,11 & 15,37 \\
1000 & 6,06 & 13,06 \\
1300 & 5,18 & 11,16 \\
1600 & 4,39 & 9,46 \\
1900 & 3,65 & 7,87 \\
\hline
\end{tabular}

En la tabla 5 se aprecia que bajo estas condiciones, se obtiene más del $10 \%$ de rendimiento térmico, siendo el salto térmico disponible mayor a medida que se disminuye la presión en el foco frío. En este sentido, la mejor condición de trabajo de entre las estudiadas sería considerar un ciclo Rankine de regeneración con el fluido de trabajo R-600a, que opere entre $3500 \mathrm{kPa}$ y $150^{\circ} \mathrm{C}$ en la entrada de la turbina, y $700 \mathrm{kPa}$ en el condensador. Con ello se obtiene un rendimiento térmico de $15,33 \%$ y se producen $7,11 \mathrm{~kW}$ de potencia eléctrica.

\section{Conclusiones}

En esta investigación se compararon las condiciones de operación para diferentes fluidos en ciclos de Rankine orgánicos, que utilizan energías de bajo grado. En cuanto al fluido R-744, este requiere de una fuente para enfriamiento que opere a temperaturas por debajo de $0^{\circ} \mathrm{C}$, por lo que no se adecúa a la situación que se quiere resolver. Al trabajar con agua de enfriamiento, correspondió al fluido R-1270 el mayor rendimiento 
térmico, cuando se compara con los demás fluidos estudiados, que presentan resultados similares entre sí. Sin embargo, no se alcanza el rendimiento térmico deseado del $10 \%$.

En cuanto a las modificaciones estudiadas para incrementar el rendimiento térmico, la que mejores resultados arrojó fue un ciclo Rankine de regeneración con el fluido de trabajo R-600a, que opere entre $3500 \mathrm{kPa}$ y $150^{\circ} \mathrm{C}$ en la entrada de la turbina, y $700 \mathrm{kPa}$ en el condensador. Este fluido, entre los estudiados, es el que permite disminuir en mayor medida la presión de trabajo en el condensador. Con estas condiciones, se obtiene un rendimiento térmico de $15,33 \%$ y se producen $7,11 \mathrm{~kW}$ de potencia eléctrica.

\section{Agradecimientos}

Los autores agradecen al Programa Conjunto de Maestría Análisis de Procesos de la Industria Química, entre la Universidad de Camagüey "Ignacio Agramonte Loynaz", Camagüey, Cuba, y la Universidad Politécnica Territorial "Alonso Gamero", Santa Ana de Coro, Estado Falcón, Venezuela.

\section{Referencias}

Air Liquide (2014). Gas Encyclopedia. Recuperado de: http://encyclopedia.airliquide.com

Bautista-Ruiz,W., Díaz-Lagos, M., \&Martínez-Ovalle, S. (2017). Caracterización de las cenizas volantes de una planta termoeléctrica para su posible uso como aditivo en la fabricación de cemento. Revista de Investigación, Desarrollo e Innovación, 8 (1), 135-146. doi: https://doi.org/10.19053/20278306. v8.n1.2017.7374

Borshanpour, B., \& Wallace, J. (2011). Organic Rankine cycle power generation for energy recovery from air compressor. 5th International Conference on Energy Sustainability, 395-413.
Campos, C., Escobar, J., Venturini, O., Silva, E., Melián, V., \& Marques dos Santos, D. (2013). Exergetic and economic comparison of ORC and Kalina cycle for low-temperature enhaced geothermal system in Brazil. Applied Thermal Engineering, 52 (1), 109-119. Recuperado de: https://www.sciencedirect.com/ science/article/abs/pii/S1359431112007259

Cano, A. (2013). Evaluación de alternativas de mejoras energéticas en centrales termoeléctricas (Tesis de grado). Cuba: ISPJAE. Recuperado de: http://tesis.cujae.edu.cu:8080/xmlui/ handle/123456789/3015

Chen, H., Yogi, D., \& Stefanakos, E. K. (2010). A review of thermodynamic cycles and working fluid for the conversion of low-grade heat. Reneweable and Sustainable Energy Reviews, 14 (9), 3059-3067.

Desintec Ingeniería S. L. (2009). Recuperación de calor para la producción de electricidad mediante ciclo orgánico de Rankine. Recuperado de: http--icaen.gencat.cat-web-.content-06_relacions_institucionals_i_comunicacio-02_agenda-actes_i_jornades-actes_i_jornades_2009-6_ desintec.pdf

Fankam, B., Papadakis, G., Lambrinos, G., \& Frangoudakis, A., (2009). Fluid selection for a low-temperature solar organic Rankine cycle. Applied Thermal Engineering, 29 (11-12), 2468-2476.

Fontalvo-Lascano, A., Romero, C. Teheran, O. Barros, P., \& Balbis-Morejon, M. (2018). Simulación termodinámica y económica de ciclos Rankine orgánicos acoplados con motores estacionarios de gas natural. 7th International Workshop Advances in Cleaner Production. 1-9.

Giuffrida, A., \& Pezzuto, D. (2017). Simulation of a scroll expander using R1233zd(E), R1234ze(Z) and their mixtures as drop-in replacements for R245fa. Energy Procedia, 129 (September), 301-306. Recuperado de: https://www.sciencedirect.com/ science/article/pii/S1876610217340262 
Lopéz, G. (2013). Análise termodinâmica de un ciclo Rankine orgânico utilizando fontes de energia renováveis (Trabajo de grado). Brasil: Universidade Federal de Itabujá. Recuperado de: http://hdl. handle.net/10016/18464

López, Y., Montes de Oca, L., Díaz, Y., Tápanez A., \& Domínguez, F. J. (2009). Simulación de un ciclo Rankine: Estudio del ciclo de vapor de una planta de ciclo combinado. Ingeniería Química, 467, 92-99.

Madhawa, H., Golubovic, M., Worek, W., \& Ikegami, Y. (2007). Optimum design criteria for an organic Rankine cycle using low-temperature geothermal heat source. Energy, 32 (9), 706-1698.

Majo, P., Chamra, L., Srinivasan, K., \& Somayaji, C. (2008). An examination of regenerative organic Rankine cycles using dry fluid. Applied Thermal Engineering, 28 (8-9), 998-1007.

Mikielewicz, J. (2010). Micro heat and power plants working in organic Rankine cycle. Polish Journal of Environmental Studies, 19 (3), 499-505. Recuperado de: https://www.researchgate.net/publication/287163662_Micro_Heat_and_Power_Plants_ Working_in_Organic_Rankine_Cycle

National Refrigerants, Inc. (2014). National Refrigerants, INC (NRI). Recuperado de: http://www. refrigerants.com

Olazabal, L. (2014). Evaluación de ciclos de potencia para la producción de electricidad a partir de sustancias orgánicas (Tesis de grado). Cuba: ISPJAE.

Pei, G., Li, J., \& Ji, J. (2010). Analysis of low temperature solar thermal electric generation using regenerative organic Rankine cycle. Applied Thermal Engineering, 30 (8-9), 998-1004. Recuperado de: https://www.researchgate.net/ publication/222135287_Analysis_of_low_temperature_solar_thermal_electric_generation_using_ regenerative_Organic_Rankine_Cycle
Quoilin, S., Van den Broek, M., Declaye, S., Dewallef, P \& Lemort, V. (2013). Techno-economic survey of organic Rankine cycle (ORC) systems. Renewable and Sustainable Energy Reviews, 22 (June), 168-186. Recuperado de: https://www.sciencedirect.com/ science/article/pii/S1364032113000592

Renedo-Estébanez, C. J. (2014). Página personal. Recuperado de: http://personales.unican.es/ renedoc

Salazar-Pereyra, M., Mora-Ortega, A., Bonilla-Blancas, A. E., Lugo-Leyte, R., \& LugoMéndez, H. D. (2017). Análisis paramétrico de las centrales geotermoeléctricas: Vapor seco, cámara flash y ciclos híbridos. DYNA, 84 (203), 273-282. doi: https://dx.doi.org/10.15446/dyna.v84n203.66126

Vásquez, R. (2013). Latinoamérica Renovable. Recuperado de: http://www.latinoamericarenovable. com

Vélez, F., Segovia, J., Martín, M., \& Antolí, G. (2012). A technical, economical and market review of organic Rankine cycle for the conversion of low-grade heat for power generation. Renewable and Sustainable Energy Reviews, 16 (6), 4175-4189. Recuperado de: https://www.sciencedirect.com/ science/article/abs/pii/S1364032112002055

Wang, X., Zhao, L., Wang, L., Zhang, W., Zhao, X., $\& W u, W$. (2010). Performance evaluation of a low temperatura solar Rankine cycle system utilyzing R 245FA. Solar Energy, 84 (10), 353-364. Recuperado de: https://www.sciencedirect.com/science/ article/abs/pii/S0038092X09002692 